\title{
Wave resistance to the movement of objects along the rocket track guides
}

\author{
Vladimir Erofeev ${ }^{1, *}$, Elena Lissenkova ${ }^{1,2}$, and Alexey Malkhanov ${ }^{1}$ \\ ${ }^{1}$ Mechanical Engineering Research Institute of RAS, Nizhny Novgorod, Russia \\ ${ }^{2}$ Nizhny Novgorod Institute of Management, Branch of RANEPA, Nizhny Novgorod, Russia
}

\begin{abstract}
The work is devoted to the study of resistance to the motion of concentrated mechanical systems along the rocket track guides, which arises as a result of the wave character of deformation of the guides. The analysis of the dependence of the wave resistance on the speed of the load is carried out.
\end{abstract}

It is known that the wave resistance to movement of loads along elastic guides is directly related to the deflection of the latter and the appearance of elastic waves. The investigations of this problem are discussed in [1-3], where constant or variable sources of forces were used as loads. Below it is considered the resistance to the movement of the load (carriage) with its own degrees of freedom, along the guide rocket track. As a guide we consider a string lying on an elastic basement. Let the motion of the carriage, which is supposed to be two elastically coupled masses $M$ and $m$, located one above the other, occurs according to the law $x=l(t)$. Assuming that the vertical displacements of the upper mass $M$ are determined by the function $y(t)$, and the vertical displacements of the lower mass are always equal to the deflections of the string $u(x, t)$ at the point of its contact $x=l(t)$ with the carriage are $u^{0}(t)$, i.e.,

$$
u^{1}(l(t), t)=u^{2}(l(t), t)=u^{0}(t) .
$$

The equations of motion of such a system look as follows:

$$
\begin{gathered}
u_{t t}-c_{0}^{2} u_{x x}+\omega_{*}^{2} u=0, \\
\ddot{y}+\Omega_{0}^{2}\left(y-u^{0}\right)=P_{0} e^{i(\Omega t+\varphi)}, \\
\ddot{u^{0}}+\omega_{0}^{2}\left(u^{0}-y\right)=\mu\left[c_{0}^{2} u_{x}+\dot{l} u_{t}\right]+Q_{0} e^{i \Omega t} \\
m \ddot{l}=-\left[\frac{1}{2} \rho u_{t}^{2}+\frac{1}{2} N u_{x}^{2}-\frac{1}{2} k u^{2}+\rho \dot{l} u_{t} u_{x}\right]+R
\end{gathered}
$$

and the conditions given at the infinity

\footnotetext{
*Corresponding author: erof.vi@yandex.ru
} 


$$
\left|u^{v}(x, t)\right|<W<\infty, x \rightarrow \pm \infty, S^{1} / h^{1}<i, S^{2} / h^{2}>i,
$$

(which guarantees the absence of any other sources, including at infinity). Here $c_{0}=$ $\sqrt{N / \rho}$ - the speed of wave propagation in a string without taking into account the basement, $\omega_{*}-$ the lowest frequency of oscillation of a string, due to the presence of an elastic basement, $\Omega_{0}=\sqrt{k_{0} / M}, \omega_{0}=\sqrt{k_{0} / m}$ - partial frequencies of oscillations of the object, determined without taking into account the influence of the string, $\mu=\rho / m, \rho-$ string density, $\Omega$ - forcing frequency, $Q_{0}=q_{01} / m, P_{0}=q_{02} / M, q_{01}, q_{02}$ - the amplitudes of the forcing forces applied to the lower and upper masses, respectively, $\varphi$ - phase shift, $S / h$ - energy transfer rate [4], $h$ - energy density, $S$ - energy flux density. The square brackets denote the difference between the limiting values, the quantities in them, to the right and left of the contact point.

Equation (3) describes the movement of the carriage along the string under the influence of an external force $\mathrm{R}$ and the longitudinal component of the reaction forces from the side of the guide (wave pressure forces) $F_{p r}=-\left[\frac{1}{2} \rho u_{t}^{2}+\frac{1}{2} N u_{x}^{2}-\frac{1}{2} k u^{2}+\rho \dot{l} u_{t} u_{x}\right]$.

Consequently, carriage that moves with a constant speed ( $i=V=$ const $)$ experiences the resistance to the movement from the elastic part of the system with a force

$$
F=-F_{p r}=\left[\frac{1}{2} \rho u_{t}^{2}+\frac{1}{2} N u_{x}^{2}-\frac{1}{2} k u^{2}+\rho V u_{t} u_{x}\right] .
$$

We will seek functions describing the steady-state forced oscillations of the system in the form $u^{v}(x, t)=A^{v} \exp \left\{i\left(\omega^{v} t-k^{v} x\right)\right\}, y(t)=B \exp (i \Omega t)$.

We obtain the following exact solution of problem (1) - (3), depending on the speed of the carriage. When $V<V_{*}=c_{0} \sqrt{1-\left(\Omega / \omega_{*}\right)^{2}}, 0 \leq \Omega<\omega_{*}$, wave numbers and frequencies of waves on the left and right of the moving source are complex. Consequently, the field of transverse displacements is localized near the carriage, and it decreases as it moves away from it

$$
\begin{gathered}
u(x, t) \\
=\left\{\begin{array}{c}
A \exp \left(-\omega_{*} \sqrt{V_{*}^{2}-V^{2}}\left(c_{0}^{2}-V^{2}\right)^{-1}(V t-x)\right) \cos \left(\Omega\left(c_{0}^{2} t-V x\right)\left(c_{0}^{2}-V^{2}\right)^{-1}+\psi_{1}\right), x<V t \\
A \exp \left(-\omega_{*} \sqrt{V_{*}^{2}-V^{2}}\left(c_{0}^{2}-V^{2}\right)^{-1}(x-V t)\right) \cos \left(\Omega\left(c_{0}^{2} t-V x\right)\left(c_{0}^{2}-V^{2}\right)^{-1}+\psi_{1}\right), x>V t
\end{array}\right. \\
A=\Delta_{A} \Delta^{-1}, \Delta_{A}=\sqrt{P_{0}^{2} \omega_{0}^{4}+2 Q_{0} P_{0} \omega_{0}^{2}\left(\Omega_{0}^{2}-\Omega^{2}\right) \cos \varphi+Q_{0}^{2}\left(\Omega_{0}^{2}-\Omega^{2}\right)^{2},} \\
\Delta=-\omega_{0}^{2} \Omega^{2}+\left(\Omega_{0}^{2}-\Omega^{2}\right)\left(-\Omega^{2}+2 \mu \omega_{*}\right) \sqrt{V_{*}^{2}-V^{2},} \\
\psi_{1}=2 \arctan \left(\frac{\Delta_{A}-Q_{0}\left(\Omega_{0}^{2}-\Omega^{2}\right)-P_{0} \omega_{0}^{2} \cos \varphi}{P_{0} \omega_{0}^{2} \sin \varphi}\right)
\end{gathered}
$$

The vibration of the upper mass of the crew is described by a function

$$
\begin{gathered}
y(t)=\Delta_{B} \Delta^{-1} \cos (\Omega t+\psi), \\
\Delta_{B}=\left\{P_{0}^{2}\left(\omega_{0}^{2}-\Omega^{2}+2 \mu \omega_{*} \sqrt{V_{*}^{2}-V^{2}}\right)^{2}+2 Q_{0} P_{0} \Omega_{0}^{2}\left(\omega_{0}^{2}-\Omega^{2}+\right.\right. \\
\left.\left.2 \mu \omega_{*} \sqrt{V_{*}^{2}-V^{2}}\right) \cos \varphi+Q_{0}^{2} \Omega_{0}^{4}\right\}
\end{gathered}
$$




$$
\psi=2 \arctan \left(\frac{\Delta_{B}-Q_{0} \Omega_{0}^{2}-P_{0}\left(\omega_{0}^{2}-\Omega^{2}+2 \mu \omega_{*} \sqrt{V_{*}^{2}-V^{2}}\right) \cos \varphi}{P_{0}\left(\omega_{0}^{2}-\Omega^{2}+2 \mu \omega_{*} \sqrt{V_{*}^{2}-V^{2}}\right) \sin \varphi}\right)
$$

We note that the amplitudes of the oscillations of the masses $\mathrm{m}$ and $\mathrm{M}$ can take both unbounded and zero values, i.e. for them in the field of parameters $V<V_{*}=$ $c_{0} \sqrt{1-\left(\Omega / \omega_{*}\right)^{2}}$ resonance and antiresonance phenomena are possible.

It follows from the above formulas that unlimited displacements are achieved at velocities

$$
V=\sqrt{V_{*}^{2}-\frac{\Omega^{4}\left(\omega_{0}^{2}+\Omega_{0}^{2}-\Omega^{2}\right)^{2}}{4 \mu^{2} \omega_{*}^{2}\left(\Omega_{0}^{2}-\Omega^{2}\right)^{2}}}<V_{*}
$$

Absence of fluctuations of the lower mass of the carriage is possible in the following cases:

a) if the upper mass of the carriage is not influenced by the transverse force $P_{0}=0$, and the frequency of the force action on the lower mass coincides with the natural oscillation frequency of the upper mass $\Omega=\Omega_{0}$.

b) the given amplitudes of the forces acting on both masses are connected with a relation $\frac{P_{0}}{Q_{0}}= \pm \frac{\Omega^{2}-\Omega_{0}^{2}}{\omega_{0}^{2}}$. The upper sign corresponds to the situation when the phase shift between forces is absent $\varphi=0$ and $>\Omega_{0}$, and the lower $-\varphi=\pi$ and $\Omega<\Omega_{0}$.

Dynamic suppression of vibrations of the upper mass of the carriage occurs, for example, if the transverse force does not act on the lower mass $Q_{0}=0$, and the movement of the carriage occurs at the following speed $V=\sqrt{V_{*}^{2}-\frac{\Omega^{4}}{4 \mu^{2} \omega_{*}^{2}}}<V_{*}$.

The deflection profile of the string is symmetrical with respect to the moving carriage with the speed $V<V_{*}$, as a result of which the constant component of the wave resistance force is zero.

In all other cases, the constant component of the resistance to the motion is different from zero. This is due to the asymmetry of the deflection profile of the guide due to the excitation of waves, whose frequencies and wave numbers are determined by formulas

$$
\omega_{1,2}=\frac{c_{0}^{2} \Omega \pm V \sqrt{V^{2} \omega_{*}^{2}-c_{0}^{2} \omega_{*}^{2}+\Omega^{2} c_{0}^{2}}}{c_{0}^{2}-V^{2}}, k_{1,2}=\frac{V \Omega \pm \sqrt{V^{2} \omega_{*}^{2}-c_{0}^{2} \omega_{*}^{2}+\Omega^{2} c_{0}^{2}}}{c_{0}^{2}-V^{2}}
$$

When $V_{*}<V<c_{0}\left(0<\Omega<\omega_{*}\right)$ there is a so-called "deceleration radiation", when a stationary source does not excite waves, since $\Omega<\omega_{*}$, but, moving with speed $V>V_{*}$, begins to excite them, experiencing deceleration effect. One of the excited waves (highfrequency) runs in front of the source, and the other (low-frequency) waves follows it. As a result, after finding the amplitudes of these waves, the deflection function will have the form:

$$
u(x, t)=\left\{\begin{array}{l}
A \cos \left(\omega_{1} t-k_{1} x+\psi_{1}\right), x<V t \\
A \cos \left(\omega_{2} t-k_{2} x+\psi_{1}\right), x>V t
\end{array}\right.
$$

Here

$$
\begin{gathered}
A=\Delta_{A} \Delta^{-1}, \Delta_{A}=\sqrt{P_{0}^{2} \omega_{0}^{4}+2 Q_{0} P_{0} \omega_{0}^{2}\left(\Omega_{0}^{2}-\Omega^{2}\right) \cos \varphi+Q_{0}^{2}\left(\Omega_{0}^{2}-\Omega^{2}\right)^{2}}, \\
\Delta=\left\{\left(\omega_{0}^{2}+\Omega_{0}^{2}-\Omega^{2}\right)^{2} \Omega^{4}+4 \mu^{2} \omega_{*}^{2}\left(\Omega_{0}^{2}-\Omega^{2}\right)^{2}\left(V^{2}-V_{*}^{2}\right)\right\}^{1 / 2},
\end{gathered}
$$




$$
\psi_{1}=2 \arctan \left(\frac{\Delta_{A} \Delta-\left(\alpha_{1} \gamma+\beta_{1} \delta\right)}{\beta_{1} \gamma-\alpha_{1} \delta}\right)
$$

Oscillations of the upper mass occur according to law

$$
y(t)=\sqrt{\alpha^{2}+\beta^{2}} \Delta^{-1} \cos (\Omega t+\psi),
$$

where $\alpha_{1}=Q_{0}\left(\Omega_{0}^{2}-\Omega^{2}\right)+P_{0} \omega_{0}^{2}\left(\Omega_{0}^{2}-\Omega^{2}\right) \cos \varphi, \beta_{1}=P_{0} \omega_{0}^{2} \sin \varphi, \gamma=-\left(\omega_{0}^{2}+\Omega_{0}^{2}-\right.$ $\left.\Omega^{2}\right) \Omega^{2}, \quad \delta=2 \mu \omega_{*}\left(\Omega_{0}^{2}-\Omega^{2}\right) \sqrt{V^{2}-V_{*}^{2}}, \quad \psi=2 \arctan \left(\frac{\sqrt{\alpha^{2}+\beta^{2}} \Delta-(\alpha \gamma+\beta \delta)}{\beta \gamma-\alpha \delta}\right), \alpha=Q_{0} \Omega_{0}^{2}+$ $\left(\omega_{0}^{2}-\Omega^{2}\right) P_{0} \cos \varphi-2 \mu \omega_{*} \sqrt{V^{2}-V_{*}^{2}} P_{0} \sin \varphi, \beta=\left(\omega_{0}^{2}-\Omega^{2}\right) P_{0} \sin \varphi+$ $2 \mu \omega_{*} \sqrt{V^{2}-V_{*}^{2}} P_{0} \cos \varphi$.

Since when $V_{*}<V$ in the system there is a radiation of energy, and, consequently, its loss, it leads to the limitation of resonant "peaks".

Substituting (5) into (4), and averaging over the oscillation frequency of the object, we obtain the expression for the constant component of the wave resistance force

$$
\langle F\rangle=\frac{\rho \Omega V \omega_{*} \sqrt{V^{2}-V_{*}^{2}} \Delta_{A}^{2}}{\left(c_{0}^{2}-V^{2}\right) \Delta^{2}} .
$$

When $0 \leq \Omega<\omega_{*}, V>c_{0}$, in contrast to the previous case, both waves run after the source, following it, and the solutions will be as follows:

$$
\begin{gathered}
u(x, t)=\left\{\begin{array}{l}
A\left\{\cos \left(\omega_{1} t-k_{1} x+\psi_{1}\right)-\cos \left(\omega_{2} t-k_{2} x+\psi_{1}\right)\right\}, x<V t \\
0, x>V t
\end{array}\right. \\
A=\Delta_{A} \Delta^{-1}, \Delta_{A}=\sqrt{P_{0}^{2} \omega_{0}^{4}+2 Q_{0} P_{0} \omega_{0}^{2}\left(\Omega_{0}^{2}-\Omega^{2}\right) \cos \varphi+Q_{0}^{2}\left(\Omega_{0}^{2}-\Omega^{2}\right)^{2}} \\
\Delta=2\left(\Omega_{0}^{2}-\Omega^{2}\right) \mu \omega_{*} \sqrt{V^{2}-V_{*}^{2}}, \psi_{1}=2 \arctan \left(\frac{P_{0} \omega_{0}^{2} \sin \varphi-\Delta_{A}}{Q_{0}\left(\Omega_{0}^{2}-\Omega^{2}\right)+P_{0} \omega_{0}^{2} \cos \varphi}\right), \\
y(t)=P_{0}\left(\Omega_{0}^{2}-\Omega^{2}\right)^{-1} \cos (\Omega t+\varphi) .
\end{gathered}
$$

We note that this also applies to the case corresponding to the Vavilov-Cherenkov effect [5], when the waves are excited by a source of zero frequency $(\Omega=0)$, that moves with with a "supercritical" speed $\left(V>c_{0}\right)$.

The average value of the wave resistance to movement force is:

$$
\langle F\rangle=\frac{\rho\left\{P_{0}^{2} \omega_{0}^{4}+2 Q_{0} P_{0} \omega_{0}^{2}\left(\Omega_{0}^{2}-\Omega^{2}\right) \cos \varphi+Q_{0}^{2}\left(\Omega_{0}^{2}-\Omega^{2}\right)^{2}\right\}}{4 \mu^{2}\left(V^{2}-c_{0}^{2}\right)\left(\Omega_{0}^{2}-\Omega^{2}\right)^{2}} .
$$

We introduce dimensionless variables:

$$
\bar{V}=\frac{V}{c_{0}}, \bar{\Omega}=\frac{\Omega}{\mu c_{0}}, \overline{\Omega_{0}}=\frac{\Omega_{0}}{\mu c_{0}}, \overline{\omega_{0}}=\frac{\omega_{0}}{\mu c_{0}}, \overline{\omega_{*}}=\frac{\omega_{*}}{\mu c_{0}}, \bar{F}=\frac{\mu^{2} c_{0}^{2}\langle F\rangle}{Q_{0}^{2} \rho} .
$$

In the Fig. 1 $(\mathrm{a}, \mathrm{b})$ there are depicted the dependences of the constant component of the resistance force of waves from the speed of movement of the carriage with different ratios between the values of the parameters $\overline{\omega_{*}}, \overline{\Omega_{0}}, \overline{\omega_{0}}$, characterizing the elastic-inertial 
properties of the string and carriage. Here for certainty $\overline{\omega_{0}}<\overline{\Omega_{0}}$, and the value of $\overline{\omega_{*}}$ varies in the plane $(\bar{V}, \bar{\Omega})$.

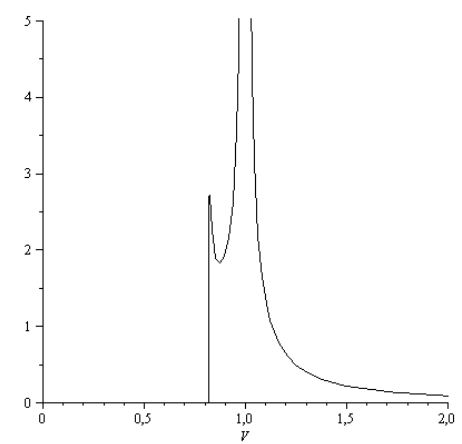

(a)

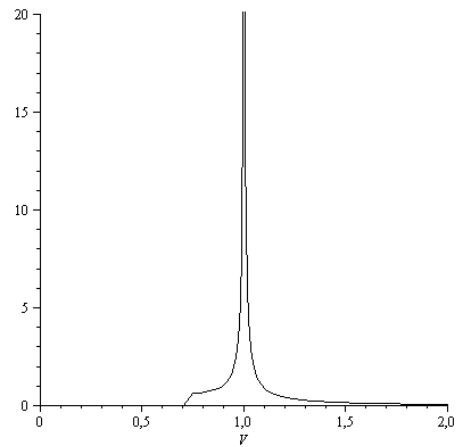

(b)

Fig. 1. Constant component of the wave resistance force, depending on the speed of the carriage for the case $\Omega<\omega_{*}$ : a) $\overline{\omega_{0}}=0.2, \overline{\Omega_{0}}=0.6, \overline{\omega_{*}}=0.4, \varphi=\pi / 6, P_{0} / Q_{0}=0.5, \bar{\Omega}=0.23$; b) $\overline{\omega_{0}}=$ $0.2, \overline{\Omega_{0}}=0.6, \overline{\omega_{*}}=0.4, \varphi=\pi / 6, P_{0} / Q_{0}=0.5, \bar{\Omega}=0.25$

For the soft basement $\omega_{*}<\sqrt{\omega_{0}^{2}+\Omega_{0}^{2}}$ there are two possibilities: $\omega_{*}<\Omega_{0}, \omega_{*}>\Omega_{0}$. If $\omega_{*}<\Omega_{0}<\sqrt{\omega_{0}^{2}+\Omega_{0}^{2}}$ at speed values $V_{*}<V<c_{0}$ there is there is a single frequency for which it is indicative that if the frequency of external influence does not exceed it, then there is a falling section (Fig. 1a) on the characteristic of the dependence of the wave resistance force on the speed of the motion of the carriage, which indicates the possible existence of instability. With the growth of the this frequency the falling section is absent in the interval $V_{*}<V<c_{0}$ (Fig. 1b). If $\Omega_{0}<\omega_{*}<\sqrt{\omega_{0}^{2}+\Omega_{0}^{2}}$, then there are two frequencies (there is no falling part on the characteristic if the frequency of the external influence falls within the interval between these frequencies). With a sufficiently rigid basement $\omega_{*}>$ $\sqrt{\omega_{0}^{2}+\Omega_{0}^{2}}$ such frequencies will be three.

In the case $\Omega>\omega_{*}$ when $0 \leq V<c_{0}$ the usual Doppler effect takes place, namely: a moving source emits one wave along its motion with frequency $\omega_{1}>\Omega$ and one in the opposite direction with frequency $\omega_{2}<\Omega$.

The increase of the speed of the carriage $V>c_{0}$ leads to the fact that both waves propagate behind the object, in the region $x<V t$, which corresponds to the so-called "complex" Doppler effect. Determining the constant component of the wave resistance force to the motion of the carriage, we obtain

\section{$\langle F\rangle$}

$=\left\{\begin{array}{c}\frac{\rho \Omega V \sqrt{V^{2} \omega_{*}^{2}+\Omega^{2} c_{0}^{2}-c_{0}^{2} \omega_{*}^{2}}\left\{P_{0}^{2} \omega_{0}^{4}+2 Q_{0} P_{0} \omega_{0}^{2}\left(\Omega_{0}^{2}-\Omega^{2}\right) \cos \varphi+Q_{0}^{2}\left(\Omega_{0}^{2}-\Omega^{2}\right)^{2}\right\}}{\left(c_{0}^{2}-V^{2}\right)\left\{\left(\Omega^{4}-\Omega^{2} \Omega_{0}^{2}-\Omega^{2} \omega_{0}^{2}\right)^{2}+4 \mu^{2}\left(\Omega_{0}^{2}-\Omega^{2}\right)^{2}\left(V^{2} \omega_{*}^{2}+\Omega^{2} c_{0}^{2}-c_{0}^{2} \omega_{*}^{2}\right)\right\}}, V< \\ \frac{\rho\left\{P_{0}^{2} \omega_{0}^{4}+2 Q_{0} P_{0} \omega_{0}^{2}\left(\Omega_{0}^{2}-\Omega^{2}\right) \cos \varphi+Q_{0}^{2}\left(\Omega_{0}^{2}-\Omega^{2}\right)^{2}\right\}}{4 \mu^{2}\left(\Omega_{0}^{2}-\Omega^{2}\right)^{2}\left(V^{2}-c_{0}^{2}\right)}, V>c_{0}\end{array}\right.$

The dependence corresponding to formula (8) is shown in Fig. 2. 


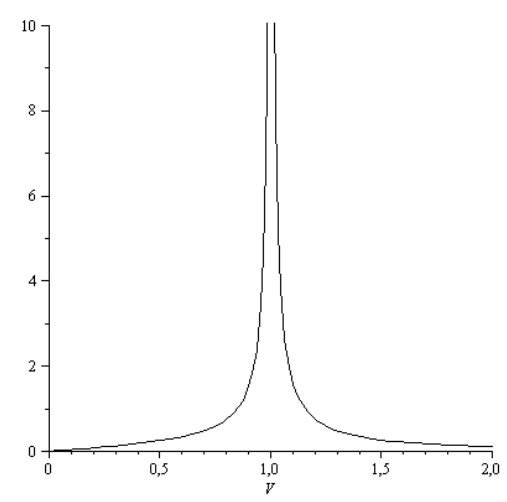

Fig. 2. Constant component of the wave resistance force, depending on the speed of the carriage for the case $\Omega>\omega_{*}$ for $\overline{\omega_{0}}=0.2, \overline{\Omega_{0}}=0.6, \overline{\omega_{*}}=1, \varphi=\pi / 6, P_{0} / Q_{0}=0.5, \bar{\Omega}=1.5$

Frequency $\Omega=\Omega_{0}$ and speed $V=c_{0}$ are resonance values, accompanied by unlimited growth of $F$. In disregard of the transverse force acting on the upper mass, at the velocities of the object $V>c_{0}$, the average value of the resistance force does not depend on the frequency of the source $\Omega$ and the natural vibration frequency of the upper mass of the carriage $\Omega_{0}$, and similarly to the resistance to movement of a load, the action of which is characterized by the transverse force.

The work was carried out within the Russian state task of the Institute of Applied Physics for conducting fundamental scientific research for 2013-2020 on the topic No. 0035-2014-0402, state registration number 01201458047 and with the support of the Russian Foundation for Basic Research (grants 17-08-01096 and 16-08-00776).

\section{References}

1. A.I. Vesnitsky, Waves in systems with moving boundaries and loads (Fizmatlit, Moscow, 2001) (in Russian)

2. V.A. Bichenkov, S.V. Krisov, V.V. Kholuyev, J. Machinery Manufacture and Reliability, 17(3), pp. 60-66 (1988)

3. V.L. Andrianov, J. Appl. Math. Mech., 57(2), pp. 156-160 (1993)

4. V.I. Yerofeyev, Ye.Ye. Lisenkova, J. Appl. Math. Mech., 77(2), pp. 230-234 (2013)

5. B.M. Bolotovskii, V.L. Ginzburg, Sov. Phys. Usp., 15, pp. 184-192 (1972) 